\title{
On the Homotopy analysis method of MSEIR epidemic model
}

\author{
${ }^{1}$ M.O. Ibrahim , ${ }^{2}$ I.I. Raji, ${ }^{2}$ A. Aladesuyi and ${ }^{3}$ A.Nwagwo \\ ${ }^{1}$ University of Ilorin, Ilorin, Kwara state, Nigeria. \\ ${ }^{2}$ The Federal Polytechnic, Ado-Ekiti, Ekiti state Nigeria. \\ Yaba College of Technology, Yaba, Lagos state, Nigeria.
}

Abstract: We considered an MSEIR epidemic model as proposed by Hethcote (2000). Homotopy analysis method is used to solve the problem because of its advantages over methods.

Keywords: Epidemic, Homotopy.

\section{Introduction}

Nonlinear equations: ordinary differential equations (ODEs) and partial differential equations (PDEs) are difficult to solve than linear especially by means of analytic methods. Traditionally, perturbation and asymptotic techniques are widely applied to obtain analytic approximations of non- linear problems in science, engineering, finance and dogreat contribution to help us understand many nonlinear phenomena. However, it is well known that perturbationmethods arestrongly dependent upon small/large physical parameters, and therefore are valid in principle only for weakly nonlinear problems.

The so-called non-perturbation techniques, such as the Lyapunov's artificial small parameter method, the $\delta$-expansion method, Adomian's decomposition method, and so on are formally dependent of small/large physical parameters. But all of these traditional non-perturbation methods cannot ensure the convergence of solution series: they are in fact only valid for weakly nonlinear problems, too. (Liao, 2004).

The Homotopy Analysis method was devised by Shijun Liao of Shanghai Jiaotong University in 1992. The Homotopy analysismethod (HAM) aims to solvenonlinear ordinary differential equations analytically. The Homotopy analysis method employs the concept of the Homotopy from topology to flexibly generate a convergent series solution for nonlinear systems. This is especially enabled by utilizing a so-called homotopyMaclaurin series to deal with the nonlinearities in the system; this series is very analogous to the better known Adomian polynomials. The method distinguishes itself from other analytical methods in four important aspects. Liao (1996), improved on HAM by introducing a non-zero parameter into the traditional way of constructing aHomotopy. He used a 2D viscous laminar flow over an infinite flat-plain governed by the nonlinear differential equation. He obtained a family of approximations which is much more general than the power series given by Blasius(as cited in Liao,1995) and can converge even in the whole region $\eta \in[0,+\infty)$.

Liao (1999) applies HAM to give an explicit, totally analytic, uniformly valid solution of the two-dimensional laminar viscous flow over a semi-infinite flat plate.

Ifidon (2009) used Homotopy analysis to the solution of the steady flow of a viscous incompressible fluid past a fixed circular cylinder. He calculated drag coefficients at 6th-order approximation and found to agree reasonably well with experimental measurements.

Liao (2004), in his paper described the basic ideas and current developments ofthe Homotopy analysis method. He also discussed some open questions and a hypothesis isput forward for future studies.

Vahdatis(2013) also considered the spread of a non-fatal disease in a population which is assumed to have constant size over the period of the epidemic.

\section{Mseir Epidemic Model}

The system of differential equations for the numbers in the epidemiology classes and the population size is 


$$
\begin{aligned}
& \frac{d M}{d t}=b(N-S)-(\delta+d) M \\
& \frac{d S}{d t}=b S+\delta M-\frac{\beta S I}{N}-d S \\
& \frac{d E}{d t}=\frac{\beta S I}{N}-(\varepsilon+d) E \\
& \frac{d I}{d t}=\varepsilon E-(\gamma+d) I \\
& \frac{d R}{d t}=\gamma I-d R \\
& \frac{d N}{d t}=(b-d N)
\end{aligned}
$$

Using the differential equation for $\mathrm{N}$, eliminating the differential equation for s by using $s=1-m-e-i-r$, $b=d+q$ and $\lambda=\beta i$.Hethcote(2000). Then the differential equations for the MSEIR model are:

$$
\begin{aligned}
& \frac{d m}{d t}=(d+q)(e+i+r)-\delta m, \\
& \frac{d e}{d t}=\lambda(1-m-e-i-r)-(\varepsilon+d+q) e, \\
& \frac{d i}{d t}=\varepsilon e-(\gamma+d+q) i, \\
& \frac{d r}{d t}=\gamma i-(d+q) r .
\end{aligned}
$$

\section{HOMOTOPY ANALYSIS METHOD (HAM)}

The homotopy analysis method (HAM) is an analytic approximation method for highly nonlinear equations in science, finance and engineering.HAM transfers a nonlinear problem into an infinite number of linear sub problems. It was first proposed by Liao in 1992 in his PhD dissertation, and modified and developed by Liao (1997).

\section{BASIC IDEA}

Consider $N[u(t)]=0$

Where $\mathrm{N}$ is a non-linear operator, $\mathrm{t}$ denotes the time, $u(t)$ is an unknown function. Let $u_{o}(t)$ denotes an initial guess of the exact solution $u(t)$ and $\mathrm{L}$ denotes an auxiliary linear operator with the property

$$
\text { Lf }=0 \quad \text { When } f=0
$$

We then construct the so-called homotopy as

$$
(1-p) L\left[\phi(t ; p)-u_{o}(t)\right]-p h H(t) N[\phi(t ; p)]=\hat{H}\left[\phi(t ; p) ; u_{o}(t), H(t), h, p\right]
$$

Where $p \in[0,1]$ is the embedding parameter, $h \neq 0$, is a non-zero auxiliary parameter, $H(t) \neq 0$ is an auxiliary function, $\mathrm{L}$ is an auxiliary linear operator. It should be emphasized that we have great freedom to choose the initial guess $u_{o}(t)$, the auxiliary linear operator $\mathrm{L}$, the non-zero auxiliary parameter $\mathrm{h}$, and the auxiliary function $H(t)$.

If homotopy (4.0) is zero, i.e

$$
\hat{H}\left[\phi(t ; p) ; u_{o}(t), H(t), h, p\right]=0 \text {. }
$$

This gives the zero-order deformation equation

$$
(1-p) L\left[\phi(t ; p)-u_{o}(t)\right]=p h H(t) N[\phi(t ; p)]
$$

Subject to the initial condition

$$
\phi(t ; p)=0
$$

When $p=0$, the zero-order deformation equation (4) becomes 
$L\left[\phi(t ; 0)-u_{o}(t)\right]=0$

$t \geq 0$

Subject to the initial condition

$$
\phi(t ; 0)=0
$$

The solution of (8.0) is

$$
\phi(t ; 0)=u_{o}(t)
$$

Also for $p=1$, since $h \neq 0, \operatorname{andH}(t) \neq 0$, the zero-order deformation equation (6.0) is equivalent to

$$
h H(t) N[\phi(t ; p)]=0, \quad t \geq 0
$$

Subject to the initial condition

$$
\begin{aligned}
& \phi(t ; 1)=0 \\
& \text { And } \quad \phi(t ; 1)=u(t)
\end{aligned}
$$

Hence, by (10) and (12), as the embedding parameter $\mathrm{p}$ increases from 0 to $1, \phi(t ; p)$ varies continuously from the initial approximation $u_{o}(t)$ to the exact solution $u(t)$. In homotopy, this is called deformation. If we expand $\phi(t ; p)$ in Taylor series with respect to $\mathrm{p}$,

$$
\phi(t ; p)=\phi(t ; 0)+\left.\sum_{n=1}^{\infty} \frac{1}{n !} \frac{\partial^{n} \phi(t ; p)}{\partial p^{n}}\right|_{p=0} p^{n}
$$

By (10), equation (14) becomes

$$
\phi(t ; p)=u_{o}(t)+\sum_{n=1}^{\infty} u_{n}(t) p^{n}
$$

Where $u_{n}(t)=\left.\frac{1}{n !} \frac{\partial^{n} \phi(t ; p)}{\partial p^{n}}\right|_{p=0}$

If the initial guess $u_{o}(t)$, the auxiliary linear operator $\mathrm{L}$, the non-zero auxiliary parameter $h \neq 0$, and the auxiliary function $H(t)$ are well chosen, so that:

i. The solution $\phi(t ; p)$ of the zero-order deformation (5.0) exists for all $p \in[0,1]$,

ii. The deformation derivative $\left.\frac{\partial^{n} \phi(t ; p)}{\partial p^{n}}\right|_{p=0}$ exists for $\mathrm{n}=1,2, \ldots$

iii. The power series (14) of $\phi(t ; p)$ converges at $\mathrm{p}=1$, then

$$
\phi(t ; 1)=u_{o}(t)+\sum_{n=1}^{\infty} u_{n}(t)
$$

This relationship provides us with a relationship between the initial guess $u_{o}(t)$ and the exact solution $u(t)$ by means of $u_{n}(t)$ for $\mathrm{n}=1,2,3, \ldots$

Next, we define thevector:

$$
\vec{U}(t)=\left\{u_{o}(t), u_{1}(t), \ldots, u_{n}(t)\right\}
$$

The governing equation and the corresponding initial condition of $u_{n}(t)$ can be deduced from the zero-order deformation equation (6) and (7). If we differentiate equations (6) and (7) $\mathrm{n}$ times with respect to the embedding parameter $\mathrm{p}$ and then setting

$\mathrm{p}=0$ and finally dividing them by $\mathrm{n}$ !, we have the so-called nth order deformation equation:

$$
L\left[u_{o}(t)-\chi_{n} u_{n-1}(t)\right]=h H(t) \Re_{n}\left(\vec{u}_{n-1}\right) .
$$

Subject to the initial condition

$$
u_{n}(t)=0
$$


Where $\mathfrak{R}_{n}\left(\vec{u}_{n-1}\right)=\left.\frac{1}{(n-1) !} \frac{\partial^{n-1} N[\phi(t ; p)]}{\partial p^{n-1}}\right|_{p=0}$

And $\quad \chi_{n}=\left\{\begin{array}{l}0 \text { when } \\ \text { 1otherwise }\end{array}\right.$

\section{Solution Of The Mseirepidemic Model By Ham}

One of the advantages of this method is that it provides a direct scheme for solving the problem.

By HAM, we choose

$$
M_{o}(t)=N_{M}, \quad E_{o}(t)=N_{E}, \quad I_{o}(t)=N_{I}, \quad R_{o}(t)=N_{R}
$$

As initial approximations of $M(t), E(t), I(t), R(t)$.

Let $p \in[0,1]$ denotes the so-called embedding parameter. The homotopy analysis method is based on a kind of continuous mappings:

$$
M(t) \rightarrow \phi_{1}(t ; p), E(t) \rightarrow \phi_{2}(t), I(t) \rightarrow \phi_{3}(t), I(t) \rightarrow \phi_{3}(t), R(t) \rightarrow \phi_{4}(t)
$$

Such that, as the embedding parameter $\mathrm{p}$ increases from 0 to $1, \phi_{j}(t), \mathrm{j}=1 \ldots 4$ varies from the initial approximation $M_{o}(t), E_{o}(t), I_{o}(t), R_{o}(t)$ to the exact solution $M(t), E(t), I(t), R(t)$. To ensure this, we choose an auxiliary linear operator as

$$
L_{j}\left[\phi_{j}(t ; p)\right]=\frac{\partial \phi_{j}(t ; p)}{\partial t}, \mathrm{j}=1,2,3,4 \text {. }
$$

With the property

$$
L_{j}\left[f_{j}\right]=0
$$

Where $f_{j}$ are integral constants. We also define the non-linear operators

$$
\begin{aligned}
& N_{1}\left[\phi_{1}(t ; p)\right]=\frac{\partial \phi_{1}(t ; p)}{d t}-(d+q)\left(\phi_{2}(t ; p)+\phi_{3}(t ; p)+\phi_{4}(t ; p)\right)+\sigma \phi_{1}(t ; p) \\
& N_{2}\left[\phi_{2}(t ; p)\right]=\frac{\partial \phi_{2}(t ; p)}{\partial t}-\lambda\left(1-\phi_{1}(t ; p)-\phi_{2}(t ; p)-\phi_{3}(t ; p)-\phi_{4}(t ; p)\right)+(\varepsilon+d+q) \phi_{2}(t ; p) \\
& N_{3}\left[\phi_{3}(t ; p)\right]=\frac{\partial \phi_{3}(t ; p)}{\partial t}-s \phi_{2}(t ; p)+(\gamma+d+q) \phi_{3}(t ; p) \\
& N_{4}\left[\phi_{4}(t ; p)\right]=\phi_{4}(t ; p)-\gamma \phi_{3}(t ; p)+(d+q) \phi_{4}(t ; p)
\end{aligned}
$$

Let $h_{j} \neq 0$ and $H_{j}(t) \neq 0 \quad \mathrm{j}=1 \ldots .4$ denotes the so-called auxiliary parameter and auxiliary function, respectively. Using the embedding parameter $\mathrm{p}$, we construct a family of equations:

$$
\begin{aligned}
& (1-p) L\left[\phi_{1}(t ; p)-M_{o}(t)\right]=p h_{1} H_{1}(t) N_{1}\left[\phi_{1}(t ; p)\right], \\
& (1-p) L\left[\phi_{2}(t ; p)-E_{o}(t)\right]=p h_{2} H_{2}(t) N_{2}\left[\phi_{2}(t ; p)\right], \\
& (1-p) L\left[\phi_{3}(t ; p)-I_{o}(t)\right]=p h_{3} H_{3}(t) N_{3}\left[\phi_{3}(t ; p)\right],
\end{aligned}
$$

$$
(1-p) L\left[\phi_{4}(t ; p)-R_{o}(t)\right]=p h_{4} H_{4}(t) N_{4}\left[\phi_{4}(t ; p)\right]
$$


As said earlier, we have great freedom to choose the auxiliary parameter $h \neq 0$, the auxiliary function $\mathrm{H}(\mathrm{t})$, the initial approximation $u_{o}(t)$ and the auxiliary linear operator $\mathrm{L}$.

When $\mathrm{p}=0$, equation (28.0) becomes

$$
\begin{aligned}
& \left.L \mid \phi_{1}(t ; 0)-M_{0}(t)\right]=0, \\
& \left.L \mid \phi_{2}(t ; 0)-E_{0}(t)\right]=0, \\
& L\left[\phi_{3}(t ; 0)-I_{0}(t)\right]=0, \\
& \left.L \mid \phi_{4}(t ; 0)-R_{0}(t)\right]=0, \\
& \quad t \geq 0
\end{aligned}
$$

Subject to the initial condition

$$
\phi_{j}(0,0)=0 \quad \mathrm{j}=1,2,3,4
$$

The solution of (30.0) and (31.0) is simply

$$
\begin{gathered}
\phi_{1}(t, 0)=M_{o}(t) \\
\phi_{2}(t, 0)=E_{o}(t) \\
\phi_{3}(t, 0)=I_{o}(t) \\
\phi_{4}(t, 0)=R_{o}(t) \ldots .
\end{gathered}
$$

When $\mathrm{p}=1$, equation (28.0) becomes

$$
h_{j} H_{j}(t) N_{j}\left[\phi_{j}(t ; p)\right]=0, t \geq 0, j=1,2, \ldots 4
$$

Subject to the initial condition

$$
\phi_{j}(t, 1)=0
$$

Since $h_{j} \neq 0, H(t) \neq 0$ and by means of the definition (27), equation (33) and (34), we have

$$
\begin{aligned}
& \phi_{1}(t, 1)=M(t), t \geq 0 \\
& \phi_{2}(t, 1)=E(t), t \geq 0 \ldots \ldots \\
& \phi_{3}(t, 1)=I(t), t \geq 0 \ldots \\
& \phi_{4}(t, 1)=R(t), t \geq 0 \ldots \ldots . . .
\end{aligned}
$$

Hence, $\phi_{j}(t, p)$ varies from initial guess $M_{o}(t), E_{o}(t), I_{o}(t), R_{o}(t)$ to the exact solution $M(t), E(t)$, $I(t), R(t)$ as the embedding parameter $\mathrm{p}$ increases from 0 to 1 .

By Taylor's theorem, we expand $\phi_{j}(t, p)$ by a power series of the embedding parameter $\mathrm{p}$ as follows:

$$
\begin{aligned}
& \phi_{1}(t, p)=M_{0}(t)+\sum_{n=1}^{\infty} M_{n}(t) p^{n}, \\
& \phi_{2}(t, p)=E_{0}(t)+\sum_{n=1}^{\infty} E_{n}(t) p^{n} \\
& \phi_{3}(t, p)=I_{0}(t)+\sum_{n=1}^{\infty} I_{n}(t) p^{n}, \\
& \phi_{4}(t, p)=R_{0}(t)+\sum_{n=1}^{\infty} R_{n}(t) p^{n},
\end{aligned}
$$

Where 


$$
\begin{aligned}
& M_{n}(t)=\left.\frac{1}{n !} \frac{\partial^{n} \phi_{1}(t ; p)}{\partial p^{n}}\right|_{p=0} \\
& E_{n}(t)=\left.\frac{1}{n !} \frac{\partial^{n} \phi_{2}(t ; p)}{\partial p^{n}}\right|_{p=0} \\
& I_{n}(t)=\left.\frac{1}{n !} \frac{\partial^{n} \phi_{3}(t ; p)}{\partial p^{n}}\right|_{p=0} \\
& R_{n}(t)=\left.\frac{1}{n !} \frac{\partial^{n} \phi_{4}(t ; p)}{\partial p^{n}}\right|_{p=0}
\end{aligned}
$$

Assume that the auxiliary parameter $h$, auxiliary function $\mathrm{H}(\mathrm{t})$, the initial approximation $M_{o}(t), E_{o}(t)$, $I_{o}(t), R_{o}(t)$ and the auxiliary operator $\mathrm{L}$ are so properly chosen that the series (36.0) converges at $\mathrm{p}=1$. Then, at $\mathrm{p}=1$, the series (36.0) becomes:

$$
\begin{aligned}
& \phi_{1}(t, 1)=M_{0}(t)+\sum_{n=1}^{\infty} M_{n}(t), \\
& \phi_{2}(t, 1)=E_{0}(t)+\sum_{n=1}^{\infty} E_{n}(t), \\
& \phi_{3}(t, 1)=I_{0}(t)+\sum_{n=1}^{\infty} I_{n}(t), \\
& \phi_{4}(t, 1)=R_{0}(t)+\sum_{n=1}^{\infty} R_{n}(t), \ldots
\end{aligned}
$$

Therefore, using equation (35), we have

$$
\begin{aligned}
& L\left[M_{n}(t)-\chi_{n} M_{n-1}(t)\right]=h_{1} H_{1}(t) \Re_{n}\left(M_{n-1}(t)\right), \\
& L\left[E_{n}(t)-\chi_{n} E_{n-1}(t)\right]=h_{2} H_{2}(t) \Re_{n}\left(E_{n-1}(t)\right), \\
& L\left[I_{n}(t)-\chi_{n} I_{n-1}(t)\right]=h_{3} H_{3}(t) \Re_{n}\left(I_{n-1}(t)\right), \\
& L\left[R_{n}(t)-\chi_{n} R_{n-1}(t)\right]=h_{4} H_{4}(t) \Re_{n}\left(R_{n-1}(t)\right),
\end{aligned}
$$

Subject to

$$
M_{0}(t)=0, E_{0}(t)=0, I_{0}(t)=0, R_{0}(t)=0 \text {. }
$$

By (Liao, 1997), we use,

$h_{j}=-1$. Using $H_{j}(t)=1$, the nth -order deformation equation (40.0) for $n \geq 1$ becomes:

$$
\begin{aligned}
& M_{n}(t)=\chi_{n} M_{n-1}(t)-\int_{0}^{t}\left[M^{1}{ }_{n-1}(\tau)-(d+q)\left(\left(E_{n-1}(\tau)+I_{n-1}(\tau)+R_{n-1}(\tau)\right)+\sigma M_{n-1}(\tau)\right)\right] d \tau \\
& E_{n}(t)=\chi_{n} E_{n-1}(t)-\int_{0}^{t}\left[E^{1}{ }_{n-1}(\tau)-\lambda\left(1-M_{n-1}(\tau)-E_{n-1}(\tau)-I_{n-1}(\tau)-R_{n-1}(\tau)\right)+(\varepsilon+d+q) E_{n-1}(\tau)\right] d \tau \\
& I_{n}(t)=\chi_{n-1} I_{n-1}(t)-\int_{0}^{t}\left[I^{1}{ }_{n-1}(\tau)-\varepsilon E_{n-1}(\tau)+(\gamma+d+q) I_{n-1}(\tau)\right] d \tau \\
& R_{n}(t)=\chi_{n} R_{n-1}(t)-\int_{0}^{t}\left[R^{1}{ }_{n-1}(\tau)-\gamma I_{n-1}(\tau)+(d+q) R_{n-1}(\tau)\right] d \tau .
\end{aligned}
$$




\section{References}

[1]. A.A.M. Arafa, S.Z. Ridaand M. Khalil, (2012). Solutions of fractional or dermodel of childhooddiseaseswithconstantvaccinationstrategy, MathematicalSciences,Letter 1(1),17-23.

[2]. E.O.Ifidon,(2009)anApplication ofHomotopyAnalysistotheViscousFlow Past aCircularCylinder. J.Appl. Math. 1-17.

[3]. F.Awawdeh,A.AdawiandZ.Mustapha,(2009).SolutionsoftheSIRmodels of epidemicsusingHAM,Chaos,SolitonsandFractals, 42,30473052.

[4]. G.A.NgwaandW.Shu,(2000).A Mathematicalmodelforendemicmalariawith variable humanand mosquito populations, Mathematicaland Computer Modeling32:747-763.

[5]. G.Shabbir, Hikhanand M.A.Sadiq,(2010).AnoteonExact Solution ofSIR AndSISepidemicmodels,arXiv:1012.5035,20-25.

[6]. H.W.Hethcote, (2000).Th e MathematicsofInfectiousDiseases,SIAMReview 42(4):599-653.

[7]. L.Yajie,B.T. Nohara,and S.J. Liao,(2010). SeriessolutionsofcoupledVan- derPolequation by means ofhomotopya nalys is method, J o u r n a l of MathematicalPhysics51:1-12. 